\title{
ESPECIE NUEVA DE ERIOSACHILA BLOW Y MANNING, 1996, (CRUSTACEA: DECAPODA), DE LA FORMACIÓN COLÓN, CUBA
}

\author{
Carlos Varela ${ }^{1}$ y Reinaldo Rojas-Consuegra ${ }^{2}$ \\ ${ }^{1}$ Acuario Nacional de Cuba (ANC), Calle 1ra \#6002 e/e 60 y 62, C. P. 11300, Playa, \\ La Habana, Cuba.carlosv@acuarionacional.cu \\ ${ }^{2}$ Museo Nacional de Historia Natural de Cuba (MNHNCu), Obispo 61, Plaza de Armas, Habana Vieja, \\ C. P. 10100, La Habana, Cuba.rojas@mnhnc.inf.cu
}

\section{RESUMEN}

Se describe una especie nueva perteneciente al género Eriosachila, hallada en la Formación Colón (Oligoceno - Mioceno), de Cuba. Esta es la segunda especie perteneciente a este género que se encuentra en estratos posteocénicos.

Palabras clave: Crustacea, Decapoda, Eriosachila, fósil, Oligoceno, Mioceno, Cuba.

\section{ABSTRACT}

A new species of fossil decapod of the genus Eriosachila is described from the Colón Formation (Oligocene - Miocene) of Cuba. This is the second species of this genus found in strata outside of the Eocene.

Key words: Crustacea, Decapoda, Eriosachila, fossil, Oligocene, Miocene, Cuba.

\section{INTRODUCCIÓN}

Aunque el conocimiento de la fauna de decápodos fósiles de Cuba haya aumentado en fecha reciente, con estudios como los de Collins et al. (2009) con material de formaciones pertenecientes al Pleistoceno Superior, así como el trabajo de Varela y Rojas-Consuegra (2009), con material de formaciones más antiguas, pertenecientes al Cretácico, al Oligoceno-Mioceno y al Plioceno, aún el conocimiento que se tiene de la misma es muy pobre.

La revisión en la colección paleontológica del Museo Nacional de Historia Natural de Cuba, nos permitió estudiar material perteneciente al género Eriosachila, el cual resultó ser una nueva especie que se describe en el presente trabajo. Los representantes del género Eriosachila son especies ya extintas que se hallan fundamentalmente en estratos del Eoceno en formaciones ubicadas en Estados Unidos, México, Panamá, San Bartolomé en el Caribe y Venezuela en Sur América (Blow y Manning, 1996; Feldmann y Schweitzer, 2004; Schweitzer y Feldmann, 2000; Schweitzer et al., 2002 y 2007). Este es el segundo hallazgo de una especie de este género en estratos posteocénicos.

\section{OBJETIVO}

- Describir una especie nueva de decápodo fósil perteneciente al género Eriosachila, encontrada en estratos posteocénicos (Oligoceno-Mioceno) de Cuba.

\section{MATERIALES Y MÉTODOS}

El material estudiado fue colectado en la cantera J4, ubicada en Jagüey Grande, provincia de Matanzas, por Lázaro William Viñola en el 2007. Esta cantera consta de calizas biodetríticas 
y margas arenosas pertenecientes a la formación geológica Colón (Brödermann, 1945), de edad Oligoceno Superior, parte alta - Mioceno Inferior. El material se encuentra depositado en la Colección Paleontológica del Museo Nacional de Historia Natural de Cuba.

\title{
RESULTADOS
}

\author{
TAXONOMÍA
}

Eriosachila cubensis sp. nov.

Figura 1, A-B

Diagnosis. Carapacho casi tan largo como ancho. Margen anterolateral con cuatro espinas. Regiones del carapacho poco desarrolladas. Margen frontal de igual ancho que el margen posterior. Región hepática no bilobada y región protogástrica sin abultamientos. Sin fisuras orbitales.

Diagnosis. Carapace almost as long as wide. Anterolateral margin with four spines. Carapace regions poorly developed. Frontal margin almost as wide as the posterior margin. Hepatic region not bilobed and protogastric region without swellings. Without orbital fissures.

Descripción del holotipo. Carapacho casi tan largo como ancho (1: 0,9), obovado, estrechándose hacia la región posterior, amplitud máxima casi en la mitad superior del carapacho, regiones pobremente definidas, regiones proto y mesogástricas, hepáticas y branquiales con abultamientos. No se observan gránulos en su superficie. Rostro no bien preservado, se extiende más allá de las órbitas y es casi el $27 \%$ del ancho máximo. Órbitas circulares, dirigidas hacia delante, no se observan fisuras orbitales, ancho frontorbital casi la mitad del ancho máximo.

Margen anterolateral fuertemente convexo, aunque no está bien preservado es posible observar someramente la base de cuatro espinas; margen posterolateral no bien preservado, margen posterior cóncavo. Región protogástrica elevada con abultamientos que se orientan paralelos al eje del carapacho. Región mesogástrica con abultamiento esférico posterior a la región protogástrica; región urogástrica no muy definida, limitada por los surcos branquicardiácos. Región cardíaca abultada. Regiones hepáticas planas, regiones branquiales alargadas, estrechas distalmente con abultamiento en su región posterior. Región ventral no bien preservada y apéndices desconocidos.

Medidas. Ancho máximo, $37 \mathrm{~mm}$; largo máximo, $33 \mathrm{~mm}$; ancho frontal, $11 \mathrm{~mm}$; ancho frontorbital, $18 \mathrm{~mm}$ y ancho posterior, $10 \mathrm{~mm}$.

Etimología. El epíteto específico se refiere a Cuba, isla en la cual se colectó el espécimen.

Tipos. Holotipo. Un ejemplar colectado en la cantera J4 en Jagüey Grande, provincia de Matanzas Cuba, por Lázaro William Viñola. Depositado en la Colección Paleontológica del Museo Nacional de Historia Natural de Cuba, MNHNCu 93. 00294.

Comentarios. El material estudiado se ha colocado tentativamente en el género Eriosachila debido la forma de su carapacho que es casi tan largo como ancho, la presencia de cuatro espinas en el margen anterolateral y la forma de las regiones en el dorso del carapacho. No obstante, nuevo material es necesario para aclarar mas detalles acerca del margen posterolateral no bien conservado.

Eriosachila cubensis, especie nueva, se distingue de E. petiti, especie tipo del género por tener espinas en su margen anterolateral y las regiones del carapacho menos desarrolladas. De E. rossi por 


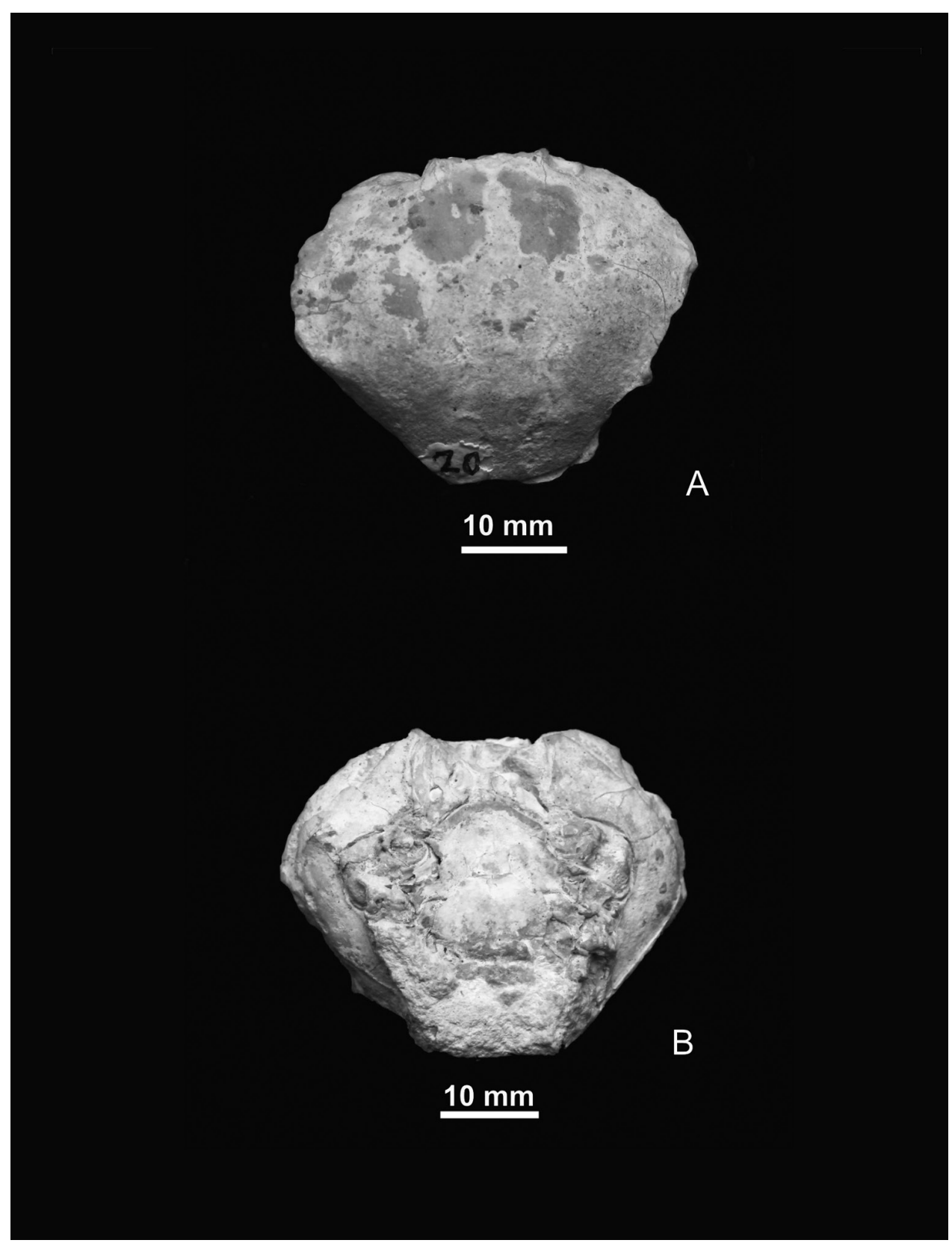

Figura 1. Eriosachila cubensis, nueva especie. A. Vista dorsal del holotipo. B. Vista ventral del holotipo.

tener el ancho del margen fontal casi igual al ancho del margen posterior. De E. bartholomaensis por no tener la región hepática bilobada ni abultamientos en la región protogástrica. De $E$. rathbunae por tener el carapacho casi tan largo como ancho, estando la amplitud máxima casi en la mitad superior del carapacho. De E. terry por tener espinas en el margen anterolateral y no tener crenulado el margen posterior. De E. orri por tener las regiones del carapacho menos desarrolladas y de E. bajaensis por no tener fisuras orbitales.

Esta es la segunda especie perteneciente al género Eriosachila que se encuentra en estratos posteocénicos, pues previamente E. rathbunae (Maury, 1930) se encontró en estratos del Mioceno de la Formación Castillo en Venezuela (Feldmann y Schweitzer, 2004). 


\section{AGRADECIMIENTOS}

A Lázaro William Viñola, un excelente recolector de gran cantidad de material fósil en la Cantera J-4, parte donada al MNHNCu. A Carrie E. Schweitzer (Kent State University), por la literatura facilitada. A Victor Isla (Acuario Nacional de Cuba) por las fotografías tomadas al material. Esta publicación se desarrolló en el marco del proyecto de investigación "Biodiversidad paleontológica del archipiélago cubano: bases cartográficas y conservacionistas”, del MNHNCu.

\section{LITERATURA CITADA}

Blow, W. C y R. B. Manning. 1996. Preliminary descriptions of 25 new decapods crustaceans from the middle Eocene of the Carolinas, U. S. A. Tulane Studies in Geology and Paleontology. 29 (1): 1-26.

Brödermann, J. 1945. Breve reseña geológica de Cuba. Revista Sociedad Cubana de Ingenieros 42 (1): 110-149.

Collins, J. S. H., R. W. Portell y S. K. Donovan. 2009. Decapod crustaceans from the Neogene of the Caribbean: diversity, distribution and prospectus. Scripta Geologica 138: 55- 111.

Feldmann, R. M. y C. E. Schweitzer. 2004. Decapod crustaceans from the lower Miocene of Northwestern Venezuela (Cerro La Cruz, Castillo Formation). Special Papers in Paleontology 71: 7-22.

Schweitzer, C. y R. M. Feldmann. 2000. New species of calappid crabs from Western North America and reconsideration of the Calappidae sensu lato. Journal of Paleontology 74 (2): 230-246.

Schweitzer, C., R. M. Feldmann, G. Gonzalez-Barba y F. J. Vega. 2002. New crabs from the Eocene and Oligocene of Baja California Sur, Mexico and an assesment of the evolutionary and paleobiogeographic implications' of Mexican fossil decapods. The Paleontological Society Memoir 76: 43 pp.

Schweitzer, C., R. M. Feldmann, G. Gonzalez-Barba y V. Cosovic. 2007. Decapod crustaceans (Brachyura) from the Eocene Tepate Formation, Baja California Sur, Mexico. Annals of Carnegie Museum 76 (1): 1-14.

Varela, C. y R. Rojas-Consuegra. 2009. Crustáceos (Deacapoda: Brachyura) fósiles de Cuba. Solenodon 8: 118-123. 